\title{
Study of the X-ray properties of blazars, based on BZCAT catalogue
}

\author{
G. M. Paronyan; A. M. Mickaelian† H. V. Abrahamyan;

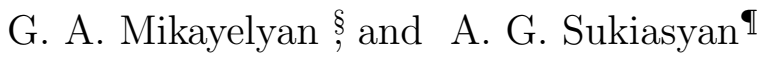 \\ NAS RA V. Ambartsumian Byurakan Astrophysical Observatory (BAO), Armenia
}

\begin{abstract}
The analysis of blazars' parameters from BZCAT leads to a conclusion that they do not have the same properties. The preliminary criterion to include an object in the catalog was the strong radio emission; however, two type of radio sources were selected: BL Lacertae (BLL) objects and Flat Spectrum Radio Quasars (FSRQ). As a number of properties are typical of blazars (strong radio emission, optical variability, continuum optical spectra, polarization, high luminosity, etc.), using the X-ray data, we investigate them to clarify which property plays the most significant role in their classification as blazars.In addition, $48 \%$ of blazars have detected radiation in X-ray have detected. We give the average statistical characteristics of blazars based on our analysis and calculations.
\end{abstract}

Keywords: active galactic nuclei, blazar, BLLac, quasar, X-ray

\section{Introduction}

Among the Active Galactic Nuclei (AGN) having SuperMassive Black Hole (SMBH), most interesting are blazars, a combination of two subtypes, BL Lac (BLL) objects and Optically Violent Variable/Highly Polarized Quasars (QSOs). A blazar is characterized as a very compact quasar associated with a presumed $\mathrm{SMBH}$ at the center of an active, giant elliptical galaxy. Blazars are probably the most energetic phenomena in the Universe. Some rare objectsmay be considered as "intermediate blazars" that have a mixture of properties from both Optically Violent Variable (OVV) or Highly Polarized Quasars (HPQ) and BLL. OVV quasars are similar to BLL but with a normal QSO spectrum (having emission lines). BLL are radio loud. HPQs have polarization typically more than $3 \%$. They are combined with OVV quasars as a single class. HPQs are made of FR II type radio galaxies. Originally,the BL Lac object has been discovered by Hoffmeister (1929) as a variable star. Later, it was identified by Schmitt (1968) with an extragalactic source.

Blazars are believed to be objects with strong relativistically beamed jets in the line of sight. It is the case when the angle between the relativistic jet axis and the line of sight is small, and the jet is Doppler boosted by a large factor and the whole spectrum (from radio to $\gamma$ - ray) is dominated by a compact,highly polarized, highly variable, superluminal, almost featureless continuum. The jet accounts for the rapid variability and compact features of both types of blazars (BLL and OVV/HPQ). The generally accepted picture is that OVV quasars are relatively powerful radio galaxies, while BLLs are relatively weak radio galaxies. In both cases, the host galaxies are giant ellipticals.

However, the definition of a blazar is still not well established. These two types have many common and different physical properties. There are many parameters that may be regarded as criteria for definition of blazars, such as high luminosity, radio flat spectrum, presence of X-ray and $\gamma$ - ray,optical and/or radio variability, polarization, etc.

*paronyan_gurgen@yahoo.com, Corresponding author

†aregmick@yahoo.com

‡abrahamyanhayk@gmail.com

§ormick@mail.ru

I andranik.suqiasyan.1995@m 
Many astronomers have made long-term observations to detect the variability of blazars. Most of the works were done for small numbers of blazars and here we compare our list with them. Below, we present the works with which we have made comparisons.

Kurtanidze et al. (2007) investigated 9 X-ray selected blazars using ST-6 CCD camera attached to the Newtonian focus of the $70 \mathrm{~cm}$ meniscus telescope in Abastumani Astrophysical Observatory.

Gupta et al. (2008) investigated five blazars using data from a $1.02 \mathrm{~m}$ optical telescope equipped with a CCD detector and BVRI Johnson broadband filters at Yunnan Astronomical Observatory, China. His observation was between October 27, 2006 and March 20, 2007. In our list,we have five variable blazars from Gupta, Alok C. et al.

Zhang et al. (2015) investigated 71 blazars (49 flat spectrum radio quasars and 22 BL Lac objects) and for the fractional variability, amplitudes of each source have been calculated in both the optical $\mathrm{R}$ band and infrared J band. In our list, we have 68 variable blazars from Bing-Kai Zhang et al.

Gaur et al. (2012) investigated 11 blazars using six optical telescopes, four in Bulgaria, one in Greece, and one in India during 2009-2011.

Hovatta et al. (2014) using Palomar Transient Factory (PTF) and the Catalina Real-Time Transient Survey (CRTS) divided 1,958 blazars to study the variability of $\gamma$ - ray.

Sandrinelli et al. (2014) investigated seven blazars using data from the Rapid Eye Mount telescope telescope located at the European Southern Observatory premises of La Silla (Chile). Light curves were gathered in the optical/near-infrared VRIJHK bands from April 2005 to June 2012.

Liu et al. (2017) investigated five blazars using many telescopes in different areas (Weihai $1 \mathrm{~m}$, Urumqi $1 \mathrm{~m}$, and Abastumani $70 \mathrm{~cm}$, University of Athens Observatory $40 \mathrm{~cm}$ ).

\section{Properties of BLAZARs}

To understand some properties of blazars, we used Roma Multifrequency Catalog of Blazars (BZCAT) 5th version (Massaro et al., 2015). Altogether, 3561 objects are given as BLL, BLQ, BLG, or BLU corresponding to BLL, FSR quasars, galaxies, and blazars of uncertain/transitional type (Table 1). BLU are adopted for sources with peculiar characteristics but also showing blazar activity: occasional presence/ absence of broad spectral lines or features, transition objects between a radio galaxy and a BLL, etc.

Table 1. Distribution of types of objects in BZCAT catalog

\begin{tabular}{|c|c|c|c|}
\hline N & Type & Number & Percentage \\
\hline 1 & BLL & 1151 & 32.3 \\
2 & BLG & 274 & 7.7 \\
3 & BLQ & 1909 & 53.6 \\
4 & BLU & 227 & 6.4 \\
5 & All & 3561 & 100 \\
\hline
\end{tabular}

In Figure 1, we give redshift distribution of BZCAT blazars (Abrahamyan et al., 2019).

In BZCAT catalog, there is information on magnitude in the $\mathrm{R}$ band and redshift. Having this, we counted absolute magnitudes for blazars using formula 1 (Véron-Cetty \& Véron, 2010).

$$
M=m+5-5 \times \log D-f(z)+\Delta m(z),
$$

where $\mathrm{D}$ is the luminosity distance as defined by Riess et al. (2004):

$$
D=\frac{c \times(1+z)}{H_{0}} \times \int_{0}^{z}\left[(1+z)^{3} \times \Omega_{M}+\Omega_{\Lambda}\right]^{-0.5} d z
$$

$\mathrm{z}$ is the redshift, $f(z)=-2.5 \times \log (1+z)^{1-\alpha}$ the $f(z)$ correction, $\Delta m(z)$ is a correction to $f(z)$ considering that the spectrum of quasars is not strictly a power law of the form $S \sim \theta^{-\alpha}$ ( $\alpha=-0.3$, Paronyan et al. (2021), Véron-Cetty \& Véron (2010)). The following values were taken for the cosmological constants in the calculations: $\Omega_{M}=0.29, \Omega_{\Lambda}=0.71$, and $H_{0}=71 \mathrm{~km} \times \mathrm{s}^{-1} / \mathrm{Mpc}$.

In Figure 2, we give graphs of the absolute magnitude versus redshift. Basically, three separated areas (BLL, BLG, and BLQ) are observed. Three types of blazars (BLL, BLG, and BLQ) are mostly separated, but there is overlap with each other to some extent. 


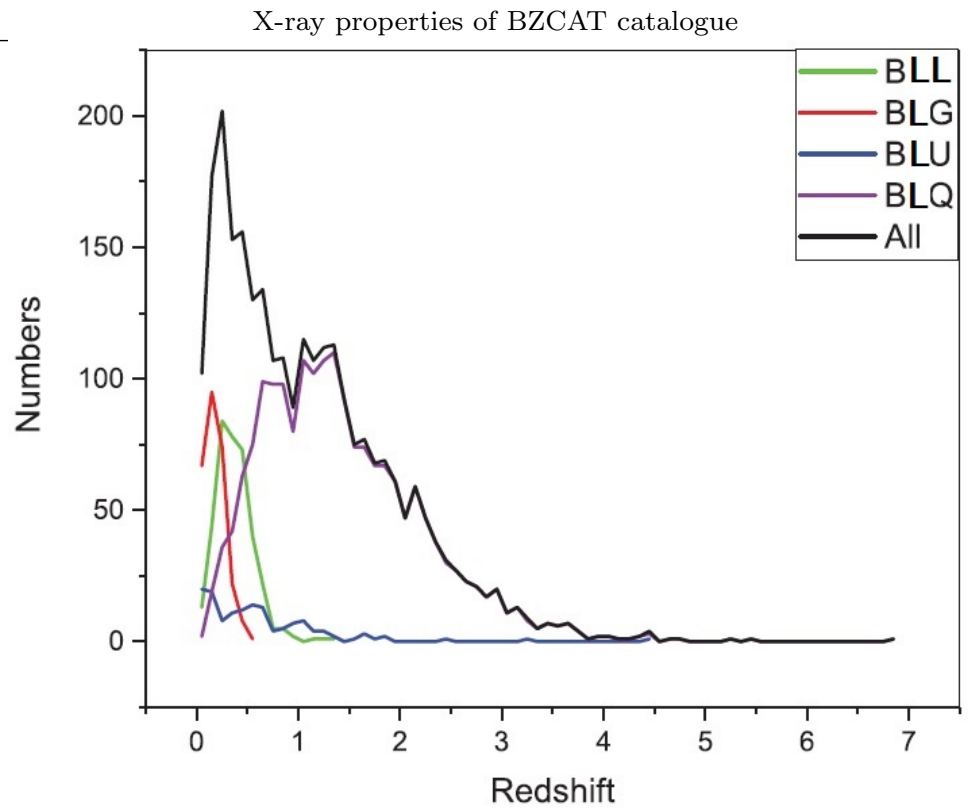

Figure 1. Redshift distribution of BZCAT blazars.
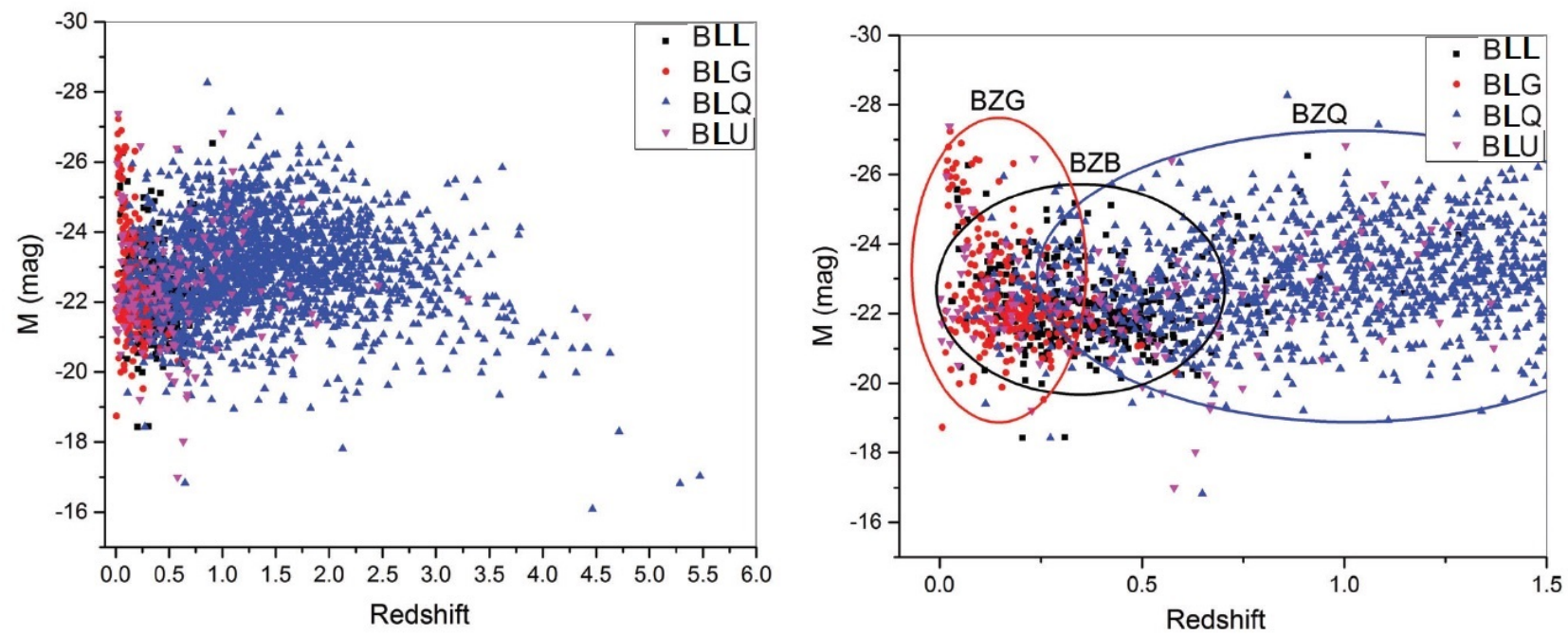

Figure 2. Absolute magnitude vs. redshift

Having the absolute magnitude, we calculated luminosities for blazars using formula 3.

$$
L=L_{\odot} \times 2.512^{M_{\odot}-M},
$$

where $L_{\odot}$ and $M_{\odot}$ are luminosity and absolute magnitude of Sun $\left(L_{\odot}=3.83 \times 10^{33} \mathrm{erg} s^{-1}, M_{\odot}=4.83\right)$. In Table 2, we give the luminosity range, average luminosity, absolute magnitude range, and average absolute magnitudes for different types of BLLs and FSRQs from the blazars catalog.

Note that the luminosity and absolute magnitude of galaxies have also contribution from the host galaxies, while BLLs and QSOs are typically pure nuclei. The numbers are approximately similar, so we do not find any significant difference between the BLL and FSRQ.

\section{Cross-correlations with X-ray catalogs and collection data}

Having 3561 blazars that have radio variability.

We cross-correlated these radio sources with X-ray catalogs: ROSAT (Boller et al., 2016), Swift-BAT (Oh et al., 2018), Chandra (Evans et al., 2010), XMM (Webb et al., 2020). In Table 3 given information of the cross-correlation X-ray catalogs.

In Table 4 information is given of the cross-correlation of optical catalogs (Abrahamyan et al., 2015). 
Table 2. Luminosity range, average luminosity, absolute magnitude range, and absolute magnitude for different types of objects from the Blazars catalog

\begin{tabular}{|c|c|c|c|c|c|}
\hline & & $\begin{array}{c}\text { Luminosity } \\
\text { range }(\mathrm{W})\end{array}$ & $\begin{array}{c}\text { Average } \\
\text { luminosity } \\
(\mathrm{W})\end{array}$ & $\begin{array}{c}\text { Absolte } \\
\text { magnitude } \\
\text { range }\end{array}$ & $\begin{array}{c}\text { Average } \\
\text { absolute } \\
\text { magnitude }\end{array}$ \\
\hline 1 & BLL & $8.05 \times 10^{28} \div 5.32 \times 10^{41}$ & $3.45 \times 10^{39}$ & $-15.09 \div-30.18$ & -23.75 \\
\hline 2 & BLG & $6.54 \times 10^{35} \div 1.38 \times 10^{39}$ & $7.85 \times 10^{37}$ & $-18.86 \div-27.08$ & -22.76 \\
\hline 3 & BLQ & $5.63 \times 10^{35} \div 7.46 \times 10^{41}$ & $4.11 \times 10^{39}$ & $-18.15 \div-30.52$ & -25.27 \\
\hline 4 & BLU & $3.58 \times 10^{30} \div 9.53 \times 10^{40}$ & $1.18 \times 10^{39}$ & $-18.16 \div-28.68$ & -23.65 \\
\hline 5 & All & $8.05 \times 10^{28} \div 7.46 \times 10^{41}$ & $3.37 \times 10^{39}$ & $-15.09 \div-30.52$ & -24.57 \\
\hline
\end{tabular}

Table 3. Identifications of blazars with X-ray catalogs

\begin{tabular}{|c|c|c|c|c|c|}
\hline Name & Band & Number of sources & Name & Band & Number of sources \\
\hline \multicolumn{7}{|c|}{ ROSAT } & \multicolumn{4}{|c|}{ XMM-NEWTON } \\
\hline$R_{b}$ & $0.1-2.0 \mathrm{kev}$ & 1590 & N1 & $0.2-0.5 \mathrm{kev}$ & 94 \\
\hline \multicolumn{7}{|c|}{ Chandra } & N2 & $0.5-1.0 \mathrm{kev}$ & 94 \\
\hline $\mathrm{b}$ & $0.5-7.0 \mathrm{kev}$ & 234 & $\mathrm{~N} 3$ & $1.0-2.0 \mathrm{kev}$ & 94 \\
\hline $\mathrm{h}$ & $2.0-7.0 \mathrm{kev}$ & 218 & $\mathrm{~N} 4$ & $2.0-4.5 \mathrm{kev}$ & 94 \\
\hline $\mathrm{m}$ & $1.2-2.0 \mathrm{kev}$ & 226 & N5 & $4.5-12.0 \mathrm{kev}$ & 94 \\
\hline $\mathrm{s}$ & $0.5-1.2 \mathrm{kev}$ & 234 & \multicolumn{3}{|c|}{ SWIFT } \\
\hline $\mathrm{u}$ & $0.2-0.5 \mathrm{kev}$ & 219 & $S_{b}$ & $14-195 \mathrm{kev}$ & 116 \\
\hline $\mathrm{w}$ & $0.1-10.0 \mathrm{kev}$ & 9 & & & \\
\hline
\end{tabular}

\section{Study of the X-ray data}

In Table 5, we give average X-ray luminosity and HR1, HR2 data for different types our blazars. From the table, we can say that, on average, BLQ is have highth luminous than BLL and BLG (BLQ > BLL > BLG). There is a difference for the values HR1 and HR2 (HR1; BLG > BLQ > BLL), (HR2; BLQ > BLG $>$ BLL).

Having X-ray luminosity, we have built the dependence of luminosity on redshift graph (Figure 3).

In Figure 3, we can see that, on average, BLG are closer than BLQ, and BLQ blazars are brighter than BLG on average.

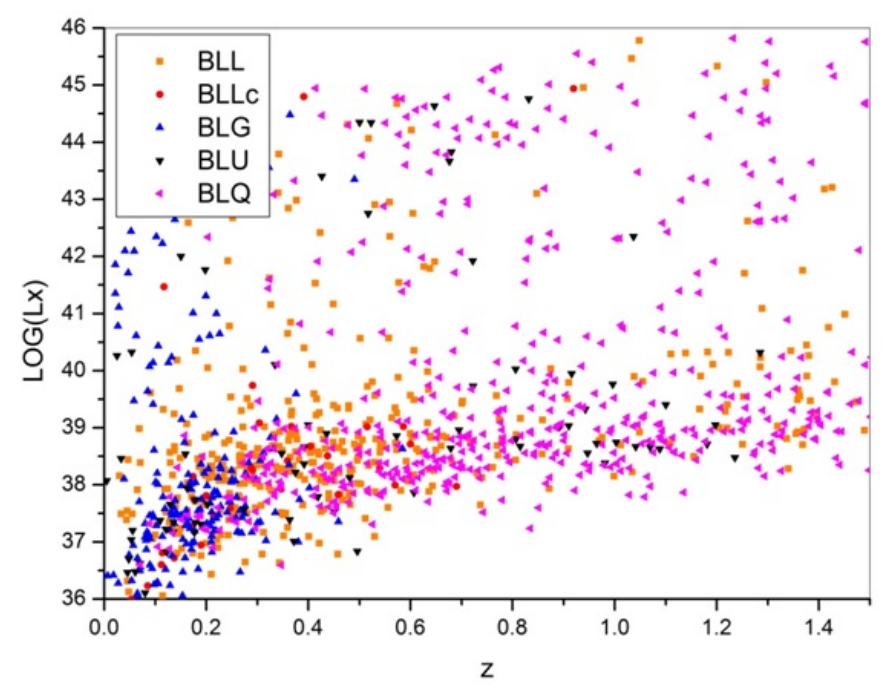

Figure 3. Distribution of redshift to X-ray luminosity BZCAT blazars.

In Table 6, we give the ratio optical luminosity to X-ray luminosity for different types blazars. From the table, we can say that, on average, BLQ is have highth value than BLL and BLG (BLQ > BLL > BLG ).

Active galaxies are very interesting objects in the Universe. In order to understand some physical properties, we must identify which properties our objects have in X-ray range. We have 1718 active galaxies 
Table 4. Optical identifications of blazars

\begin{tabular}{|c|c|c|c|c|}
\hline N & Catalogs & Epoch & Photobands & Identified objects \\
\hline 1 & APM & POSS1 & b,r & 1977 \\
\hline 2 & USNO A2.0 & POSS1 & B1, R1 & 3151 \\
\hline 3 & USNO B1.0 & POSS1/POSS2 & B1, R1/B2, R2 & 3492 \\
\hline 4 & GSC 2.3 .2 & POSS2 & F, j & 3501 \\
\hline 5 & SDSS DR16 & & $\mathrm{u}, \mathrm{g}, \mathrm{r}, \mathrm{i}, \mathrm{z}$ & 1446 \\
\hline
\end{tabular}

Table 5. X-ray Luminosity range, average luminosity, HR1 range, and HR2 range for different types of objects from the Blazars catalog

\begin{tabular}{|c|c|c|c|c|c|}
\hline & & $\begin{array}{c}\text { Luminosity } \\
\text { range }(\mathrm{W})\end{array}$ & $\begin{array}{c}\text { Average } \\
\text { luminosity }(\mathrm{W})\end{array}$ & $\begin{array}{c}\text { Average } \\
\text { HR1 range }\end{array}$ & $\begin{array}{c}\text { Average } \\
\text { HR2 range }\end{array}$ \\
\hline 1 & BLL & $8.90 \times 10^{31} \div 1.45 \times 10^{46}$ & $8.89 \times 10^{43}$ & 0.218 & 0.151 \\
\hline 2 & BLG & $1.51 \times 10^{35} \div 3.01 \times 10^{44}$ & $2.83 \times 10^{42}$ & 0.415 & 0.199 \\
\hline 3 & BLQ & $3.89 \times 10^{36} \div 1.07 \times 10^{47}$ & $1.05 \times 10^{45}$ & 0.328 & 0.203 \\
\hline 4 & BLU & $9.02 \times 10^{30} \div 5.72 \times 10^{44}$ & $1.89 \times 10^{43}$ & 0.434 & 0.177 \\
\hline 5 & All & $9.02 \times 10^{30} \div 1.07 \times 10^{47}$ & $5.31 \times 10^{44}$ & 0.308 & 0.181 \\
\hline
\end{tabular}

with X-ray fluxes at different wavelengths. A very important X-ray property for objects is the X-ray spectral index. It shows steep spectra. Using more bands, we have developed a graph for all galaxies ( $\lg [$ flux] vs. $\lg$ [frequencies]). Using an $\lg [$ flux] versus $\lg$ [frequencies] graph for each source, we have made linear fitting. The software "Origin" gives the formula for each linear fit, and using that, we have measured the X-ray spectral index for each source. The plot shows steep spectra for each line, and that is considered spectral index. As examples, we give average X-ray spectra for our objects in Figure 4.

In Figure 4, average X-ray spectra for different types of our objects are given. It is obvious that BLQ on average, have steeper X-ray spectra than BLL, BLU and BLGs (Table 7).

Table 7 illustrates the average information of spectral indices.

Using information from Table 7, we can see that BLL and BLG have the same spectra on average, but BLQ has steeper spectra than BLL and BLG.

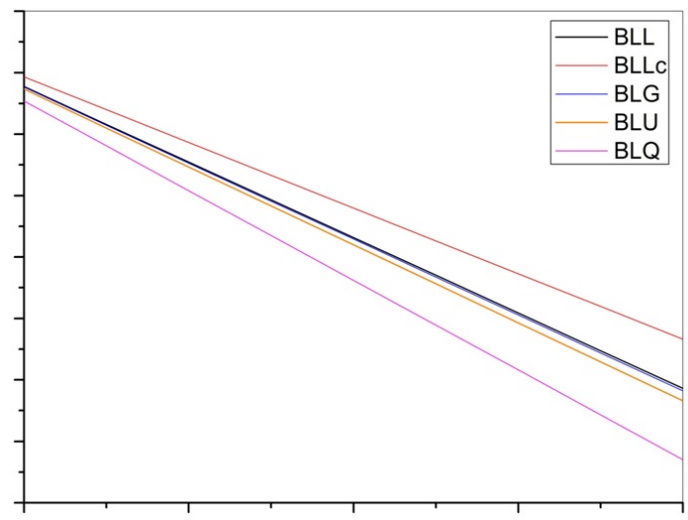

Figure 4. Distribution of X-ray spectral index BZCAT blazars.

\section{Summary and Conclusion}

We have analyzed flux and luminosities for 1572 blazars (ROSAT 0.1-2.0 kev band) $\left(L_{B L L}=8.89 \times 10^{43}\right.$ $\left.\mathrm{W}, L_{B L G}=2.83 \times 10^{42} \mathrm{~W}, L_{B L Q}=1.05 \times 10^{45} \mathrm{~W}, L_{B L U}=1.89 \times 10^{43} \mathrm{~W}, L_{B L L c}=4.69 \times 10^{43} \mathrm{~W}\right)$.

We have analyzed absolute magnitudes and luminosities for 3117 blazars (SDSS DR16 r band) ( $M_{B L L}$ $=-23.75, M_{B L G}=-22.76, M_{B L Q}=-25.27, M_{B L U}=-23.65, M_{B L L c}=-22.58, L_{B L L}=3.45 \times 10^{39} \mathrm{~W}, L_{B L G}$ $\left.=7.85 \times 10^{37} \mathrm{~W}, L_{B L Q}=4.11 \times 10^{39} \mathrm{~W}, L_{B L U}=1.89 \times 10^{37} \mathrm{~W}, L_{B L L c}=3.14 \times 10^{38} \mathrm{~W}\right)$.

We analyzed X-ray spectral indices for 1572 blazars $\left(\alpha_{B L L}=-3,075, \alpha_{B L L c}=-3,054, \alpha_{B L G}=-3,095\right.$, $\left.\alpha_{B L U}=-3,168, \alpha_{B L Q}=-3,659\right)$. On average, BLL and BLG have the same X-ray spectra, whereas BLQ has 
Table 6. Distribution of dependence SDSS r band luminosity to ROSAT X-ray luminosity for Blazars (Lg $(\operatorname{Lr} / \mathrm{Lx}))$

\begin{tabular}{|c|c|c|c|c|}
\hline N & Type & Min & Max & Average \\
\hline 1 & BLL & -7.35 & 2.16 & -1.09 \\
2 & BLG & -6.89 & 3.39 & -0.71 \\
3 & BLQ & -7.84 & 1.96 & -1.58 \\
4 & BLU & -6.84 & 2.31 & -0.83 \\
\hline
\end{tabular}

Table 7. Distribution of X-ray spectral index

\begin{tabular}{|c|c|c|}
\hline $\mathbf{N}$ & Type & X-ray index \\
\hline 1 & BLL & -3.075 \\
2 & BLG & -3.095 \\
3 & BLQ & -3.659 \\
4 & BLU & -3.168 \\
\hline
\end{tabular}

steeper X-ray spectra than BLL and BLG.

We have estimated physical sizes for our objects. That objects of various activity types statistically have the same average sizes. However, due to small number of objects, this result is statistically not substantiated; hence, we need further studies using bigger numbers of objects to follow the real differences in distributions of physical sizes for different types of Blazars.

\section{Acknowledgements}

The work was supported by the Science Committee of RA, in the frames of the research project No. 21AG-1C053" Revelation of the early stages of galaxy evolution by means of multiwavelength study of active galaxies".

\section{References}

Abrahamyan H. V., Mickaelian A. M., Knyazyan A. V., 2015, Astronomy and Computing, 10, 99

Abrahamyan H. V., Mickaelian A. M., Paronyan G. M., Mikayelyan G. A., 2019, Astronomische Nachrichten, 340,437

Boller T., Freyberg M. J., Trümper J., Haberl F., Voges W., Nandra K., 2016, Astron. Astrophys. , 588, A103

Evans I. N., et al., 2010, Astrophys. J. Suppl. Ser. , 189, 37

Gaur H., et al., 2012, Mon. Not. R. Astron. Soc. , 425, 3002

Gupta A. C., Fan J. H., Bai J. M., Wagner S. J., 2008, Astron. J. , 135, 1384

Hoffmeister C., 1929, Astronomische Nachrichten, 236, 233

Hovatta T., et al., 2014, Mon. Not. R. Astron. Soc. , 439, 690

Kurtanidze O. M., Nikolashvili M. G., Kimeridze G. N., Sigua L. A., Kapanadze B. Z., 2007, in Karas V., Matt G., eds, Vol. 238, Black Holes from Stars to Galaxies - Across the Range of Masses. pp 399-400, doi:10.1017/S1743921307005649

Liu X., et al., 2017, Mon. Not. R. Astron. Soc. , 469, 2457

Massaro E., Maselli A., Leto C., Marchegiani P., Perri M., Giommi P., Piranomonte S., 2015, Astrophys. Space. Sci. , 357, 75

Oh K., et al., 2018, Astrophys. J. Suppl. Ser. , 235, 4

Paronyan G. M., Mickaelian A. M., Abrahamyan H. V., Mikayelyan G. A., 2021, Astrophysics, 64, 277

Sandrinelli A., Covino S., Treves A., 2014, Astron. Astrophys. , 562, A79

Schmitt J. L., 1968, Nature. , 218, 663

Véron-Cetty M. P., Véron P., 2010, Astron. Astrophys. , 518, A10

Webb N. A., et al., 2020, Astron. Astrophys. , 641, A136

Zhang B.-K., Zhou X.-S., Zhao X.-Y., Dai B.-Z., 2015, Research in Astronomy and Astrophysics, 15, 1784 